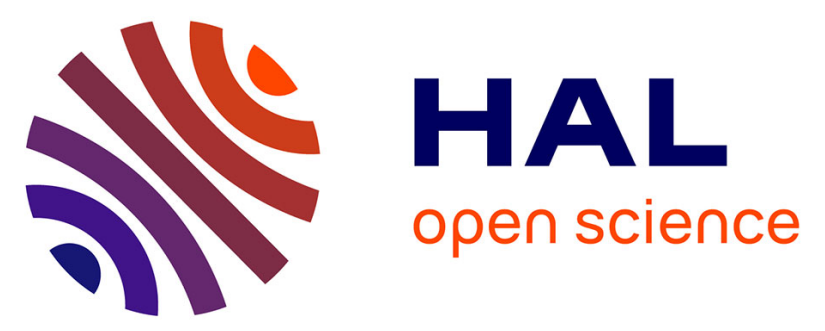

\title{
Evolution of the properties of helium nanobubbles during in situ annealing probed by spectrum imaging in the transmission electron microscope
}

\author{
K. Alix, M.-L. David, J. Dérès, C. Hébert, Laurent Pizzagalli
}

\section{To cite this version:}

K. Alix, M.-L. David, J. Dérès, C. Hébert, Laurent Pizzagalli. Evolution of the properties of helium nanobubbles during in situ annealing probed by spectrum imaging in the transmission electron microscope. Physical Review B: Condensed Matter and Materials Physics (1998-2015), 2018, 97 (10), 10.1103/PhysRevB.97.104102 . hal-02114782

\section{HAL Id: hal-02114782 \\ https://hal.science/hal-02114782}

Submitted on 29 Apr 2019

HAL is a multi-disciplinary open access archive for the deposit and dissemination of scientific research documents, whether they are published or not. The documents may come from teaching and research institutions in France or abroad, or from public or private research centers.
L'archive ouverte pluridisciplinaire HAL, est destinée au dépôt et à la diffusion de documents scientifiques de niveau recherche, publiés ou non, émanant des établissements d'enseignement et de recherche français ou étrangers, des laboratoires publics ou privés. 


\title{
Evolution of the properties of helium nanobubbles during in situ annealing probed by spectrum imaging in the transmission electron microscope
}

\author{
K. Alix, ${ }^{1}$ M.-L. David,,${ }^{1, *}$ J. Dérès, ${ }^{1}$ C. Hébert,${ }^{2}$ and L. Pizzagalli ${ }^{1}$ \\ ${ }^{1}$ Departement of Physics and Mechanics of Materials, Institut P', CNRS-Université de Poitiers UPR 3346, \\ SP2MI, BP 30179, F-86962 Futuroscope Chasseneuil Cedex, France \\ ${ }^{2}$ CIME, EPFL-SB-CIME-GE, CH-1015 Lausanne, Switzerland
}

(Received 9 December 2017; published 7 March 2018)

\begin{abstract}
The evolution of nanometric helium bubbles in silicon has been investigated using spatially resolved electron energy-loss spectroscopy during in situ annealing in the transmission electron microscope. This approach allows the simultaneous determination of both the morphology and the helium density in the bubbles at each step of the annealing. Structural modification and helium emission from bubbles of various diameters in the range 7.5 to $20 \mathrm{~nm}$ and various aspect ratios of 1.1 to 1.9 have been studied. We clearly show that helium emission takes place at temperatures where bubble migration had hardly started. At higher temperatures, the migration (and coalescence) of voids is clearly revealed. For helium density lower than $150 \mathrm{He} \mathrm{nm}^{-3}$, the Cerofolini's model taking into account the thermodynamical properties of an ultradense fluid reproduces well the helium emission from the bubbles, leading to an activation energy of $1.8 \mathrm{eV}$. When bubbles exhibit a higher initial helium density, the Cerofolini's model fails to reproduce the helium emission kinetics. We ascribe this to the fact that helium may be in the solid phase and we propose a tentative model to take into account the properties of the solid.
\end{abstract}

DOI: 10.1103/PhysRevB.97.104102

\section{INTRODUCTION}

Helium is one of the most inert elements in nature [1] and its solubility in most materials is very low. Its presence in materials may thus result from implantation, irradiation [2], or pressureinduced helium trapping $[3,4]$ for instance. In the former case, the energetic helium atoms induce the displacement of a large number of atoms from their lattice position creating excess defects (self-interstitials and vacancies) that may agglomerate to form larger clusters and/or may interact with the implanted atoms as well. In many materials, the interaction of vacancies and helium leads to the formation of bubbles that may modify the mechanical and physical properties of materials. In particular, bubbles being responsible for the embrittlement of materials [5], the study of helium bubbles in metal and metallic alloys, nanostructured or not, is a very active field in the nuclear domain, for instance, see Refs. [6-10]. Further, it has been recently suggested that helium bubbles themselves, could be used to control the damage evolution in irradiated materials, because they can act as a sink for point defects [9].

In covalent materials, helium bubbles are formed following high fluence implantation as well and the idea to transform bubbles from a liability into an asset has also emerged twenty years ago in the semiconductor field. The most known application is probably the formation of helium and hydrogen bubbles, the platelets, used to produce silicon-on-insulator wafers (SOI) [11]. However, other potential applications have been demonstrated such as annihilation of dislocations in GaN [12], reducing the threading dislocation density via the enhancement of the strain relaxation of $\mathrm{SiGe} / \mathrm{Si}$ heterostructures [13],

\footnotetext{
*marie-laure.david@univ-poitiers.fr
}

the proximity gettering of metallic impurities [14], or the fabrication of ultrathin buried oxide layers in silicon [15]. Finally, low-energy helium plasma treatments have recently received considerable interest for nanostructuration of surfaces with applications in domains of energy conversion and storage devices, by both bottom-up [16] or top-down approaches $[17,18]$. In particular, nanopores in amorphous silicon coatings produced using such methods have been revealed to contain a high helium density [19].

For all these applications, the control of the bubble formation and evolution is essential. This requires an in-depth understanding of the underlying physical mechanisms. The rough picture of bubble formation and evolution in a covalent material like silicon is as follows. When implanted at high fluence, helium leads to the formation of tiny bubbles (about $2 \mathrm{~nm}$ in diameter) homogeneously distributed in a layer, the depth of which being controlled by the implantation energy. During annealing, the bubbles evolve into larger bubbles, helium is emitted from the bubbles, which transform into voids [20], stable up to high temperatures [21]. This is somewhat different from what is observed in metals where helium dissociation from bubbles is effectively negligible and for which bubble coarsening processes are dominated by the thermal migration of bubbles and subsequent coalescence [22].

However, although this scenario is generally admitted, the understanding of the structural evolution of the bubbles and especially its correlation with the helium content is still missing. On the one hand, the release of helium during annealing has been investigated by thermal helium desorption spectroscopy (THDS) [20,21,23,24]. On the other hand, the dynamics of cavity coarsening during high-temperature annealing has been studied mainly by transmission electron microscopy [21,25-29] but also by GISAXS (grazing incidence small angle 
x-ray scattering) for instance [30,31]. In theory, combining these experiments could allow following the structural and chemical evolution of the bubbles. However, as the implantation conditions [32-34] and the annealing procedure [35-37] play a crucial role in the bubble formation and evolution, the relevance of a comparison between different experiments is seriously limited.

The simultaneous monitoring of both the morphology of the bubbles and the helium content would allow to overcome these limitations. For instance, spatially resolved electron energy-loss spectroscopy (EELS) has been shown to be a powerful tool to study in situ the irradiation-induced resolution of helium [38,39] in the transmission electron microscope (TEM). Unfortunately, in silicon, the technique is destructive and can not be used to study thermal emission. We have recently developed a spatially resolved EELS approach based on energy-filtered transmission electron microscopy-spectrum imaging (EFTEM-SI) acquisition of the data avoiding helium detrapping under the electron beam and allowing the investigation of a large number of bubbles [40,41]. EFTEM-SI thus appears as a powerful tool to unravel the detail of the evolution of bubbles during thermal annealing as it offers the unique possibility to investigate simultaneously the structural and the chemical modifications of the bubbles during an in situ annealing for instance.

In this paper, we have examined the structural and chemical modifications of the individual bubbles of a bubble layer created by high fluence helium implantation in silicon using in situ EFTEM-SI experiments. The paper is organized in four parts. First, experimental conditions are detailed. The experimental results are reported in a second part and the helium emission is modeled in a third part. Finally, the results are discussed. Note that in this paper, the word "cavity" is used to refer to both a bubble (containing helium) and a void, which does not contain helium.

\section{EXPERIMENTS}

P-type silicon samples were implanted at room temperature with helium at high fluence and moderate energy $(50 \mathrm{keV}$, $7.5 \times 10^{16} \mathrm{~cm}^{-2}$, and $0.01 \mathrm{Am}^{2}$ ). The samples were then annealed at 773 or $973 \mathrm{~K}$ for $30 \mathrm{~min}$ under vacuum in a tubular furnace; they are respectively labeled S773K and S973K.

The investigation of both the helium emission from individual bubbles and their structural modification was conducted through in situ annealing and spatially resolved EELS in the TEM. For that purpose, cross-sectional TEM thin foils were prepared by mechanical polishing down to $10 \mu \mathrm{m}$ and ion milling in a GATAN-PIPS apparatus at low energy $(2.5 \mathrm{keV}$ Ar) and low incidence $\left( \pm 8^{\circ}\right)$. To minimize irradiation damage, a final step was performed at $\pm 4^{\circ}$ for two minutes. The experiments were performed using a JEOL 2200FS with FEG operated at $200 \mathrm{keV}$, equipped with an in-column $\Omega$ filter and a Gatan Ultrascan $2048 \times 2048$ pixel CCD camera. Prior to data acquisition, the sample was oriented to minimize diffraction contrast in the observed area and an objective aperture was inserted giving a collection semiangle for the spectrometer of $5.65 \mathrm{mrad}$. A nominal $10^{5} \times$ magnification, resulting in a final $200 \times 200 \mathrm{~nm}^{2}$ field of view on the CCD, was chosen in order to observe ten or more of the largest bubbles simultaneously.

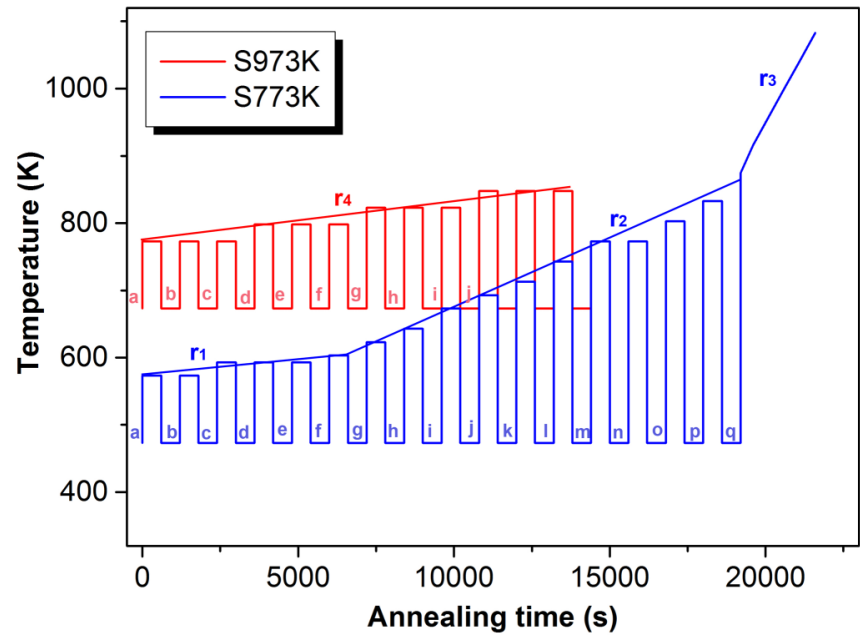

FIG. 1. Temperature ramps used during the in situ annealing in the TEM, in red for the S973K sample and in blue for the S773K sample. The letters refer to the helium density maps shown in Figs. 3 and 6.

The choice of the zone to be measured is critical: the bubble superposition has to be as limited as possible in order to measure individual bubbles. Our criterion was that the central area of each bubble does not overlap with any part of another. Besides, the chosen area has to include as many as possible filled bubbles. These areas were about 40-nm thick for the S973K sample and $25 \mathrm{~nm}$ thick for the S773K sample. Note that during TEM sample preparation, some bubbles can be cut and emptied, while still retaining their appearance in conventional TEM. Helium chemical mapping by EFTEM was then performed prior to the in situ annealing experiments to determine if an area with low superposition contains a majority of helium-filled bubbles.

The in situ annealing was performed using a GATAN heating sample holder. For both samples, the same methodology was applied: the annealing was performed using annealing steps of $10 \mathrm{~min}$, followed by datacube acquisition at a lower temperature, 473 and $673 \mathrm{~K}$, respectively, for the S773K and S973K samples. These temperatures were chosen to avoid any helium detrapping during the datacube acquisition (about 20 min per datacube) and to minimize thermal cycling and the associated spatial drift. The $\mathrm{S} 773 \mathrm{~K}$ sample was annealed from 573 to $1123 \mathrm{~K}$ and the $\mathrm{S} 973 \mathrm{~K}$ sample from 773 to $823 \mathrm{~K}$. These ranges were chosen owing to preliminary in situ annealing experiments during which only a zero-loss and a He $K$-edge filtered micrographs (no spectrum images) were acquired. The annealing steps used for each sample are shown in Fig. 1. To model the experimental data, the annealing steps were fitted with linear laws. For the S773K sample, the first part of the annealing from $573 \mathrm{~K}$ to $600 \mathrm{~K}$ was quasi-isothermal with a ramp, $r_{1}$ of $0.0062 \mathrm{~K} \mathrm{~s}^{-1}$. A second ramp, $r_{2}$ of $0.0395 \mathrm{~K} \mathrm{~s}^{-1}$ was used from 600 to $830 \mathrm{~K}$ and a final faster annealing ramp, $r_{3}$ of $0.083 \mathrm{~K} \mathrm{~s}^{-1}$ was used from 823 to $1123 \mathrm{~K}$ once helium was almost totally emitted. During this last temperature ramp, only underfocused energy-filtered images were recorded. The S973K sample annealing was modeled as a unique ramp, $r_{4}$ of $0.013 \mathrm{~K} \mathrm{~s}^{-1}$. 
The datacubes were acquired in two steps between -3 and $32 \mathrm{eV}$ using $0.2-\mathrm{eV}$ energy increments between energy planes and a slit of $1 \mathrm{eV}$. The elastic peak was first acquired with a 0.5 seconds exposure time and the second part of the datacube $(10-32 \mathrm{eV})$ was recorded with a $5 \mathrm{~s}$ exposure time. For each sample, two zones were analyzed after each annealing step, i.e., two data cubes were recorded. However, it turned out that the data recorded in one of the two regions of the S773K sample were not analyzable, leading to poor statistics as compared to the S973K sample.

The realignement of the datacubes was realized using a homemade procedure, allowing the combined correction of the nonisochromaticity and spatial drift [40]. The He $K$ edge was extracted after median filtering, multiple scattering deconvolution and fit of the plasmons of the $\mathrm{SiO}_{2} / \mathrm{Si}$ interface, the bulk $\mathrm{Si}$ and the cavity by Gaussian or pseudo-Voigt functions. The acquisition and treatment procedures of the spectrum images are described in further detail in Ref. [40]. The helium density, $n_{\mathrm{He}}$, is determined through its relation with the blueshift of the He $K$ edge, $\Delta E$, using

$$
n_{\mathrm{He}}=\frac{1}{C_{\mathrm{He}}} \Delta E,
$$

where $\Delta E=E-E_{0}[38,42,43] . E$ is the energy of the He $K$ edge measured in the bubble under study. $E_{0}$ is the energy of the He $K$ edge for the free atom. In the literature, a value $E_{0}=$ $21.218 \mathrm{eV}$ [44] determined by absorption experiments was systematically used $[38,45,46]$. However, as we have discussed in Ref. [40], using this value could lead to an overestimation of the helium density. Here, we thus take $E_{0}=21.71 \mathrm{eV}$, which corresponds to the energy position of the He $K$ edge for nearly empty bubbles as explained in Sec. IV. Moreover, $C_{\mathrm{He}}$ in Eq. (1) is assumed to be $0.015 \mathrm{eV} \mathrm{nm}^{3}$ as determined by previous experiments in similar systems [38].

After each annealing step and prior to any EFTEM-SI acquisition, underfocused $(-1000 \mathrm{~nm})$ zero-loss energy-filtered images were acquired. Quantitative analysis of these images were performed to determine both the aspect ratio and size of the bubbles. The method used is based on a standard image threshold procedure which has proven to be very successful to analyze embedded nanoparticles [47]. It, however, results in an error on the bubble size which is due to the thresholding process and to the thickness of the Fresnel fringe used to image the bubbles. This error is estimated at $\pm 1 \mathrm{~nm}$. The bubble aspect ratio is defined as $a / b$ with $a$ the largest dimension. In the following, the bubble diameter is defined as the diameter of a disk having the same area as an ellipse of parameter $a$ and $b$.

\section{EXPERIMENTAL RESULTS}

The initial state of the samples has been reported in detail in Ref. [40]. Before in situ annealing, the bubble layer is composed of small bubbles homogeneously distributed in the layer, labeled $S$ bubbles, and of rafts of larger bubbles located at the deepest edge of the bubble layer, labeled $L$ bubbles (Fig. 2). The bubbles of the S973K sample are almost spherical with a mean aspect ratio of 1.1. Their mean diameter ranges from

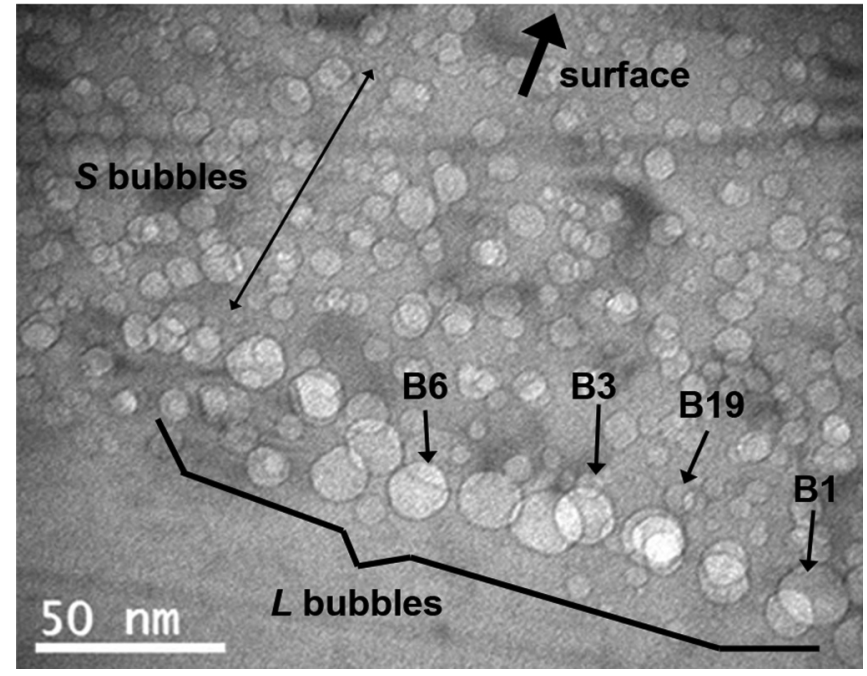

FIG. 2. Zero-loss energy-filtered micrograph (1-eV slit) in underfocus conditions $(-1000 \mathrm{~nm})$ of the $7.5 \times 10^{16} \mathrm{He} \mathrm{cm}^{-2}, 50 \mathrm{keV}$ implanted silicon sample annealed at $700^{\circ} \mathrm{C}, 30 \mathrm{~min}$ (S973K sample). The emission kinetics of the bubbles labeled B1, B3, B6, and B19 are shown in Fig. 3.

$9 \mathrm{~nm}$ for the $S$ bubbles to $20 \mathrm{~nm}$ for the $L$ ones. The initial helium density in most of the analyzed bubbles is in the range 70 to $100 \mathrm{He} \mathrm{nm}^{-3}$.

The helium density maps acquired during in situ annealing are shown in Fig. 3. Maps of the other analyzed region can be seen in Ref. [49]. The helium emission during in situ annealing was monitored from 49 bubbles, with diameters ranging from 7.5 to $20 \mathrm{~nm}$. As soon as annealing starts $(10 \mathrm{~min}$ at $773 \mathrm{~K})$, helium is emitted from the bubbles. The kinetics of helium emission seems to be faster for some of the bubbles. For instance, the bubble labeled B3 in Fig. 3(a) is almost empty after the two first steps of annealing, whereas the others still contain helium. At temperatures higher than $823 \mathrm{~K}$, all bubbles are almost empty. From these maps, the helium density was measured in the center of each bubble (averaged over several pixels) as a function of the annealing time. For the sake of clarity, the emission kinetics of only four typical bubbles are shown in Fig. 3(k). The bubbles labeled B1, B3, and B6, exhibit similar characteristics before in situ annealing. They are respectively 19,17 , and $16.6 \mathrm{~nm}$ in diameter and are all located in the raft of $L$ bubbles, with an initial helium density of about $97 \mathrm{He} \mathrm{nm}^{-3}$. As seen, the helium emission kinetics from these three bubbles are similar although slightly faster for B3 and B6 than for B1. The helium emission kinetics from smaller bubbles (7-10 $\mathrm{nm}$ in diameter) follows the same trend. For instance, the emission kinetics of bubble B19 $(8.8 \mathrm{~nm}$ in diameter with an initial helium density of $80 \mathrm{He} \mathrm{nm}^{-3}$ ) is shown in Fig. 3(k).

The evolution of the mean aspect ratio and of the mean equivalent diameter of both $S$ and $L$ bubble populations (respectively small and large symbols) during the in situ annealing is shown in Fig. 4. As seen, these characteristics stay almost constant, although a small decrease of the mean equivalent diameter can be noticed for both $S$ and $L$ bubbles.

The bubble layer analyzed in the S773K sample is shown in Fig. 5(a). Focusing first on the bubbles in the $L$ layer, twelve 

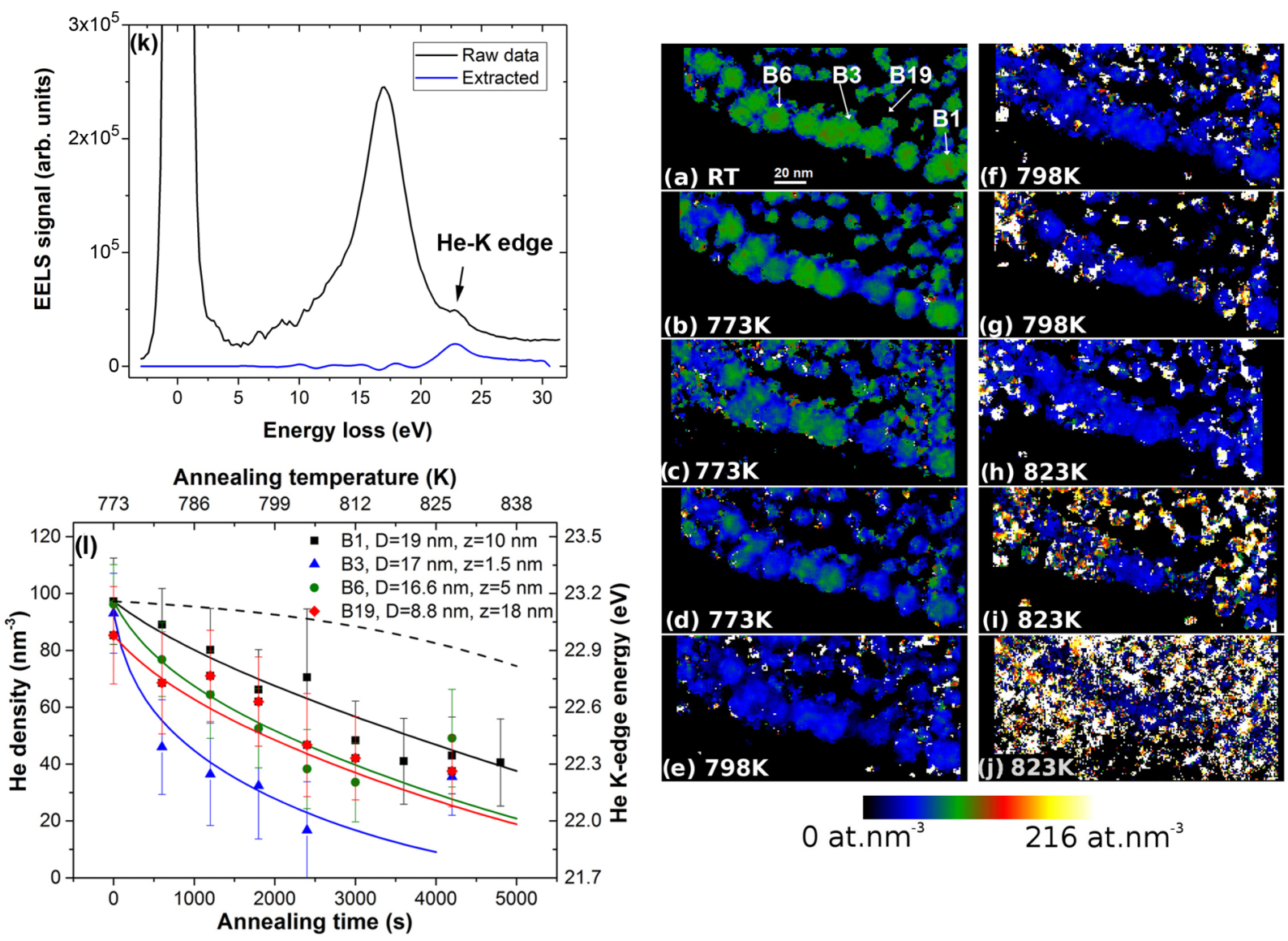

FIG. 3. [(a)-(j)] Helium density maps for the same region of the S973K sample as shown in Fig. 2 as a function of the in situ annealing temperature and time indicated in Fig. 1. All the spectrum images were acquired at $673 \mathrm{~K}$. (k) shows in black a typical EEL spectrum in a bubble (B3) after an annealing at $773 \mathrm{~K}$ during $10 \mathrm{~min}$ [image (b)], averaged over $7 \times 7$ pixels and in blue the extracted He- $K$ edge following the procedure detailed in Ref. [40]. (1) Helium density measured in the center of bubbles B1, B3, B6, and B19. The symbols represent the experimental data, the solid lines show the Cerofolini's model for each bubble, and the black dashed line shows the first-order kinetics model for B1. $D$ is the equivalent diameter measured on the TEM micrograph and $z$ is the depth from the surface at which the bubble is located as determined using the Cerofolini's model. The activation energy $E^{\text {act }}$ was fixed at $1.8 \mathrm{eV}$ to fit the data.

bubbles, labeled B1-B12, were analyzable. The other bubbles of this layer do not contain helium as seen on the He $K$ edge filtered image, Fig. 5(b), of the same region as Fig. 5(a). They probably have been cut during the sample preparation. The morphology of B1-B12 bubbles is characterized by a broad distribution of both the equivalent diameter in the range 7 to $16 \mathrm{~nm}$ and the aspect ratio in the range 1.2 to 1.9 , the smallest bubbles being the more spherical. The morphological evolution of the bubbles during the in situ annealing is depicted in Figs. 5(e) and 5(f). As seen, the aspect ratio of the bubbles tends to become homogeneous. The morphology of the bubbles, which were already nearly spherical at room temperature, does not change, whereas the aspect ratio of the other bubbles decreases continuously to approximately 1.1-1.2. Such an aspect ratio, close to unity, corresponds in fact to the lowest energy equilibrium, tetra-kaidechaedral shape, typical of voids in silicon $[29,48]$. This value is reached at $950 \mathrm{~K}$ and does not change during further annealing. This is particularly clear for bubble B9 with the highest initial aspect ratio [red stars in Fig. 5(f)]. Furthermore, the equivalent diameter of the bubbles stays constant during the annealing.
Above about $910 \mathrm{~K}$, a motion of cavities is observed. Some cavities migrate towards the surface (B2 for instance at $910 \mathrm{~K}$ ) or in the thin foil (B6 and B10 at $970 \mathrm{~K}$ or B5 and B9 and $1050 \mathrm{~K}$ ) and an event of migration and coalescence is clearly observed (B3+B4 at 910 K). The movie included in Ref. [49] allows for a better viewing of the phenomena [49]. Those lead to a decrease of the density of bubbles as seen in Fig. 5(d). Note that some of the initially empty bubbles_-voids - are lost at the surface from $850 \mathrm{~K}$.

We have not performed any quantitative analysis of the morphology of the $S$ bubbles in this sample. Indeed, the density of bubbles is very high [see Fig. 5(a)], the bubble diameter is small, about $4 \mathrm{~nm}$, and no quantitative analysis of the He $K$ edge was possible as evidenced by the helium chemical map [Fig. 5(b)] where helium-filled $S$ bubbles are hardly distinguishable. It is, however, worth noticing that migration towards the surface and migration and coalescence of the $S$ cavities start at about $920 \mathrm{~K}$, leading to a smaller density of larger bubbles 5 to $6 \mathrm{~nm}$ in diameter.

The helium density maps acquired during in situ annealing are shown in Figs. 6(a)-6(q) for bubbles B1 to B12 of the 


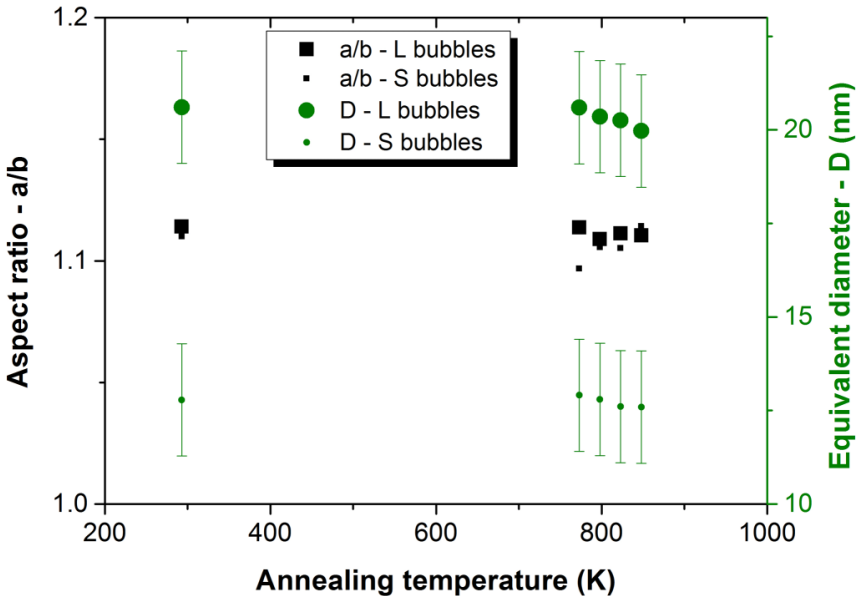

FIG. 4. Mean aspect ratio $a / b$ in black and mean equivalent diameter $D$ in green for the $S$ and $L$ bubbles (respectively the large and small symbols) as a function of the annealing temperature. The average is realized over 49 bubbles from both the region shown in Fig. 2 and another region shown in Ref. [49].

S773K sample. The helium density measured in each bubble is reported in Figs. 6(r)-6(s). For the sake of completeness, a typical EEL spectrum (in black) and the extracted He $K$ edge following the procedure detailed in Ref. [40] (in blue) are shown in Fig. 7. This spectrum has been acquired in the bubble B8 after the first step of annealing (573 K, $10 \mathrm{~min}$ ). Before annealing, most bubbles exhibit a helium density of about $180 \mathrm{He} \mathrm{nm}^{-3}$. However, three bubbles, B2, B9, B11, exhibit a smaller initial helium density of $120 \mathrm{He} \mathrm{nm}^{-3}$. Like the S973K sample, helium emission is observed since the first steps of the annealing irrespective of the initial helium density. At $833 \mathrm{~K}$, helium can still be detected in some of the bubbles, but for higher temperatures, all the bubbles are almost empty. Except for bubbles B2 and B11 for which a low helium density is reached very rapidly, helium emission from bubbles occurs in two steps: a first one during the first part of the annealing, nearly isothermal from 570 to $590 \mathrm{~K}$ and a second step between 670 and $840 \mathrm{~K}$, until the bubbles are almost empty. Finally, it is worth noticing that we did not observe any He atom exchange between the bubbles.

\section{HELIUM DETRAPPING KINETICS MODELING}

The helium emission from the bubbles is first modeled following the approach of Cerofolini et al. [20,50]. The helium effusion is driven by the difference in helium chemical potential between the bubble and the matrix. From the partition function of a lattice gas made of an assembly of He interstitial atoms distributed on tetrahedral sites [51,52], the chemical potential of $\mathrm{He}$ in the $\mathrm{Si}$ matrix is derived. For $\mathrm{He}$ in the bubble, following the approach used in [52], we considered the ultradense fluid statistical physical description proposed by Cerofolini et al. [20,53] to determine the chemical potential. Indeed, the initial helium density in the bubbles, of the order of $100 \mathrm{He} \mathrm{nm}{ }^{-3}$ is very high, it is thus not possible to describe the thermodynamic behavior of helium using neither the ideal gas nor the van der Waals gas law. The ultradense fluid model is built from a van der Waals model, in which
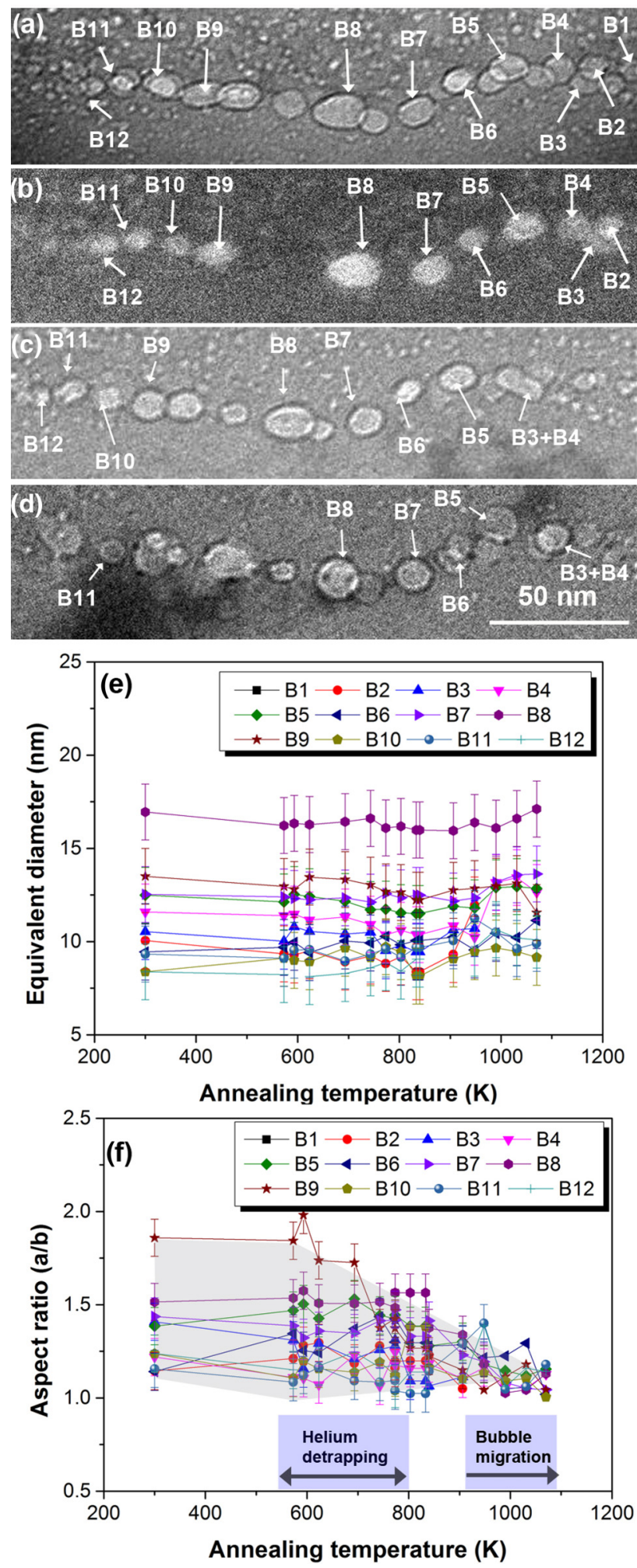

FIG. 5. (a) Zero-loss energy-filtered micrograph (1-eV slit) in underfocus conditions $(-1000 \mathrm{~nm})$ of the S773K sample $(7.5 \times$ $10^{16} \mathrm{He} \mathrm{cm}^{-2}, 50 \mathrm{keV}$ implanted silicon sample annealed at $500{ }^{\circ} \mathrm{C}$, $30 \mathrm{~min}$ ) before any in situ annealing. (b) Energy-filtered image on the He $K$ edge $(24.2 \pm 1 \mathrm{eV})$ extracted from a spectrum image acquired on the same region as (a). (c) and (d) Same region and same imaging conditions as (a) after respectively an in situ annealing at 910 and 1133 K. (e) Equivalent diameter and (f) aspect ratio of the bubbles labeled B1 to B12. 

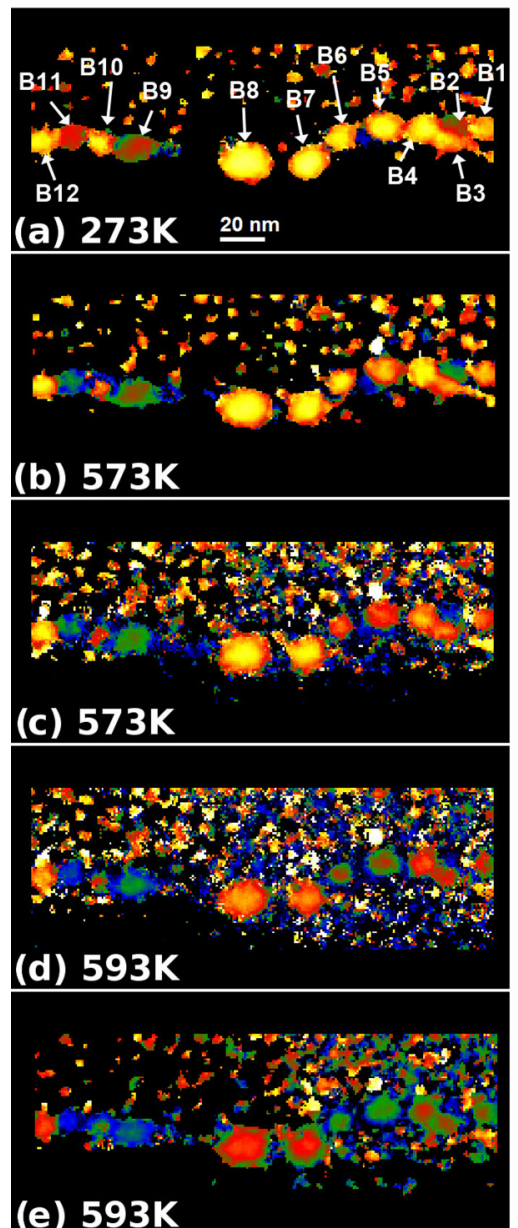

(e) $593 \mathrm{~K}$

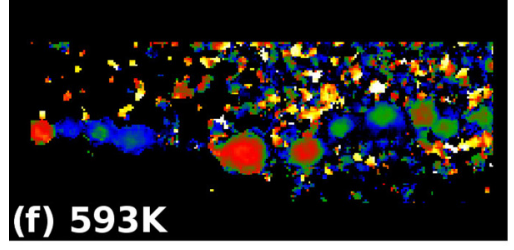

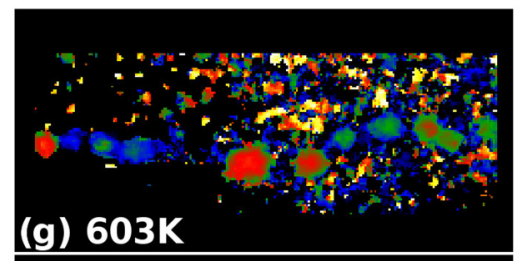
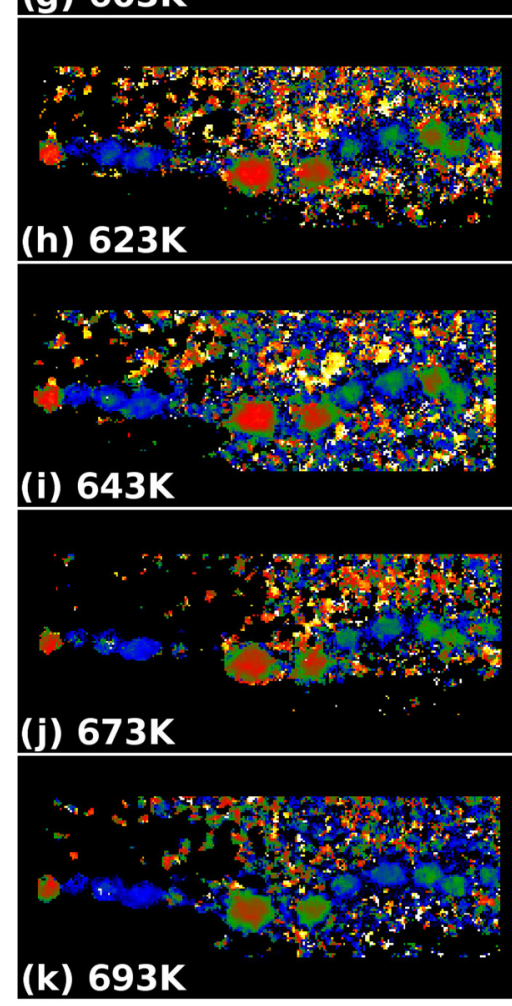

(k) 693K

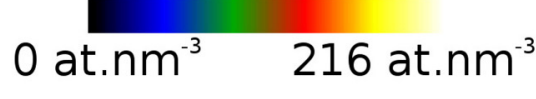

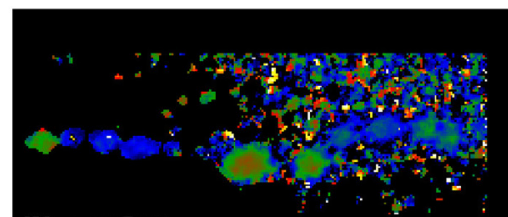

\section{(I) $713 \mathrm{~K}$}

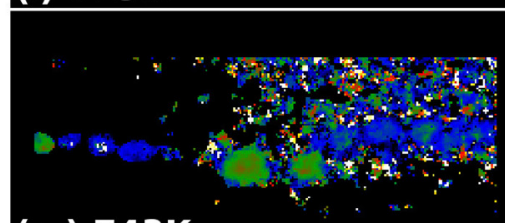

\section{(m) 743K}

\section{(n) $773 \mathrm{~K}$}

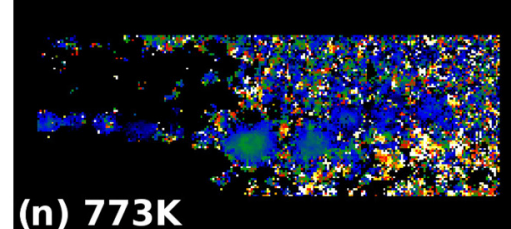

\section{(o) $773 \mathrm{~K}$}

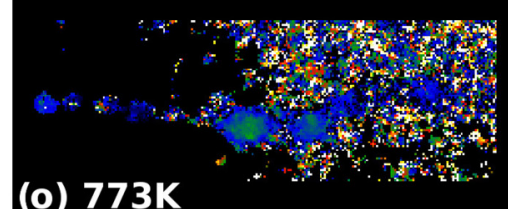

\section{(p) $803 \mathrm{~K}$}
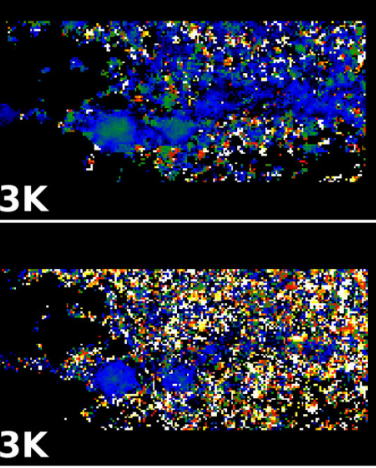
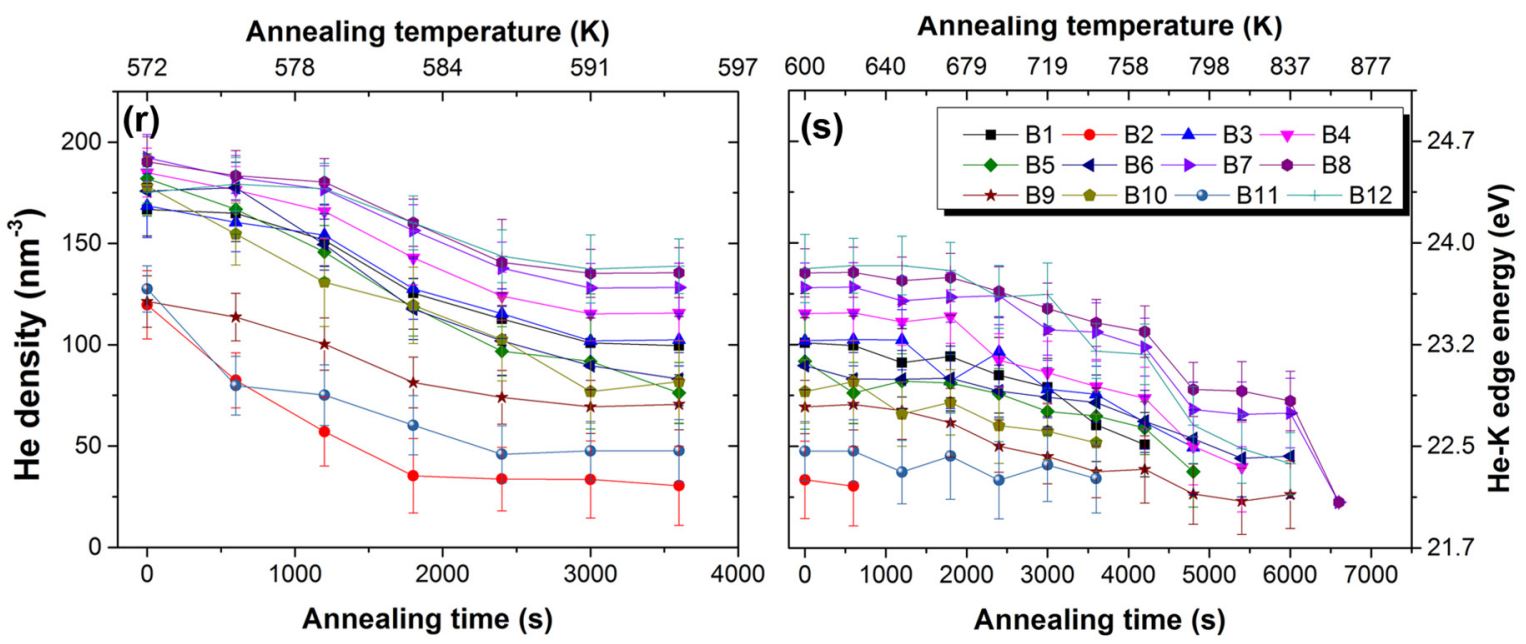

FIG. 6. [(a)-(q)] Helium density maps for the same region of the S773K sample as shown in Fig. 5 as a function of the in situ annealing temperature and time indicated in Fig. 1. All the spectrum images were acquired at $473 \mathrm{~K}$. [(r)-(s)] Helium density measured in the center of bubbles B1-B12 and averaged over several pixels. 


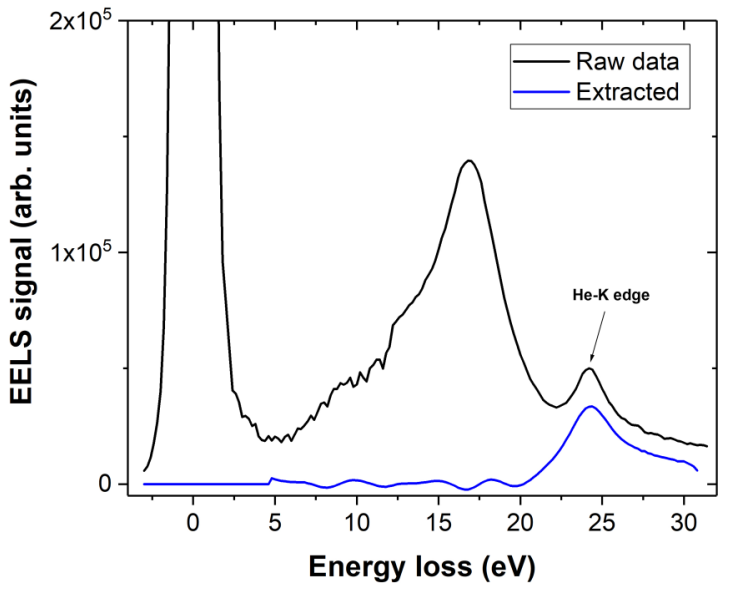

FIG. 7. Typical EEL spectrum in a bubble of the S773K sample (B8) after an annealing at $573 \mathrm{~K}$ during $10 \mathrm{~min}$ [Fig. 6(b)] averaged over $7 \times 7$ pixels in black and in blue the extracted He $K$ edge following the procedure detailed in Ref. [40].

finite-size effects are taken into account by associating a defined constant volume to each particle (the covolume), and by including the interaction energy between all particles. To allow for a better description of the very high particle density, the covolume should depend on the particle density and on the temperature in the ultradense fluid model. A simplified repulsive potential is used to model the interactions between particles $[U=u(r)=w \exp (-r / \chi)$, with $w=3000 \mathrm{eV}$ and $\chi=0.192 \AA]$ [20]. With this model, the free energy of the total system and the chemical potential of the He atoms are derived. Next, assuming equilibrium between the lattice gas in the Si matrix and the ultradense fluid bubble allows to specify the helium density in the bubble as a function of the helium density outside. The rate equation describing the variation of $n_{\mathrm{He}}$ is then given by

$$
\begin{aligned}
-\frac{d n_{\mathrm{He}}}{d t}= & 2 \frac{D_{0} c_{s} \lambda_{T}^{3}}{z V}\left(\frac{\exp \left(-h v_{i} / 2 k_{B} T\right)}{1-\exp \left(-h v_{i} / k_{B} T\right)}\right)^{3} \\
& \times \frac{n_{\mathrm{He}}}{1-b\left(n_{\mathrm{He}}, T\right) n_{\mathrm{He}}} \exp \left(\frac{b\left(n_{\mathrm{He}}, T\right) n_{\mathrm{He}}}{1-b\left(n_{\mathrm{He}}, T\right) n_{\mathrm{He}}}\right) \\
& \times \exp \left(-\frac{E^{\mathrm{act}}-1 / 2 \omega u\left(k n_{\mathrm{He}}^{-1 / 3}\right)}{k_{B} T}\right) .
\end{aligned}
$$

$D_{0}$ is the prefactor of the Arrhenius expression for the diffusivity of helium in interstitial sites in $\mathrm{Si}[D=$ $\left.D_{0} \exp \left(-\epsilon_{i} /\left(k_{B} T\right)\right)\right], \lambda_{T}=h / \sqrt{2 \pi m k_{B} T}$ is the thermal de Broglie wavelength (with $h$ the Planck constant and $m$ the helium atom mass), $v_{i}$ is the vibration frequency of helium as an interstitial in the Si lattice (assumed to be the same for the three normal modes), $k_{B}$ is the Boltzmann constant, $b\left(n_{\mathrm{He}}, T\right)$ is the dynamical covolume given by [20]

$$
\begin{aligned}
b_{T}(T) & =\frac{2}{3} \pi\left[\rho \ln w /\left(\frac{3}{2} k_{B} T\right)\right]^{3}, \\
b_{D}\left(n_{\mathrm{He}}\right) & =\frac{2}{3} \pi\left[k\left(n_{\mathrm{He}}^{-1 / 3}-\rho \ln \omega\right)^{3}\right], \\
b\left(n_{\mathrm{He}}, T\right) & =b_{T} b_{D} /\left(b_{D}+b_{T}\right) .
\end{aligned}
$$

$c_{s}$ is the concentration of interstitial sites in the bubble region. The repulsive energy of an atom in the field of the $\omega$ neighboring atoms is taken into account through the term $\frac{1}{2} U=\frac{1}{2} \omega u\left(k n_{\mathrm{He}}^{-1 / 3}\right)$, where $k$ is related to the He packing factor in the bubble $(k=1.122$ for the face-centered-cubic structure [20]). $z$ is equal to the depth from the surface at which the cavity layer is located (the deepest edge of the bubble layer). In Ref. [20], this parameter is set on the basis of TEM micrographs or Monte Carlo simulations with SRIM for instance [54]. In our case, the closest surface is not the implanted surface but the two surfaces of the TEM thin foils. The determination of the position of a buried defect in the TEM thin foil is a long standing problem [55]. For the sake of simplicity, the bubble is here assumed to lie in the middle of the thin foil. Thus the factor 2 in Eq. (2) takes into account that the loss of helium may occur from both surfaces and $z$ is left as a floating parameter. It is important to note that the surface is not necessarily flat as it may contain holes left by bubbles cut during the sample preparation, i.e., $z$ is not necessarily equal for two close bubbles. $V$ is the volume of the bubble per unit area. It is determined based on the TEM micrographs of the explored region. Finally, $E^{\text {act }}$ corresponds to the activation energy for $\mathrm{He}$ emission when the chemical potential of the He atoms in the bubble is null; it is taken equal to $1.8 \mathrm{eV}$ as determined previously $[20,24,52,56]$.

This model is first applied to the emission kinetics of the S973K sample in order to determine $E_{0}$, the position of the He $K$ edge at low helium density. As seen in Fig. 3(1), the He emission kinetics from the bubbles of this sample are characterized by a single mechanism, well reproduced by the Cerofolini's model (solid lines). Focusing first on bubbles B1, B3, and B6, which exhibit similar morphology and initial helium density, it is clear that the difference in the He emission kinetics is due to the position of the bubble with respect to the thin foil surfaces ( $z$ parameter), the closer the surface, the stronger the emission. The dependence on the bubble volume through the term $V$ in the rate equation [Eq. (2)] is seen with bubble B19, which is deeper from the surface but is also smaller than B6, leading to a similar emission kinetics than B6.

The comparison between the Cerofolini's model and the first-order effusion rate equation suggested by Griffioen $e t$ al. and often used in the literature $[23,24,31,57]$ to describe helium effusion from bubbles [black dashed line in Fig. 3(k)], clearly emphasized that finite-size effects should be taken into account when dealing with a dense fluid.

To determine $E_{0}$, the emission kinetics of the ten largest bubbles were modelled with $E_{0}$ and $z$ as floating parameters, $n_{\mathrm{He}}$ being given by Eq. (1). An average of the $E_{0}$ value was made, leading to a value of $21.71 \mathrm{eV} \pm 0.02 \mathrm{eV}$. This value is used in the course of the paper to determine the helium density through Eq. (1). Note that the error of $0.02 \mathrm{eV}$ is the standard deviation of the set of data values determined on the ten bubbles.

We now focus on the bubbles in the S773K sample and start to discuss the emission kinetics of bubbles characterized by the highest initial He density (all bubbles except B2, B9, and B11). These clearly exhibit a two steps process. The first one occurs during the nearly isothermal annealing up to $2500 \mathrm{~s}$ and 587 $\mathrm{K}$ above which the second step starts. We found that the first 

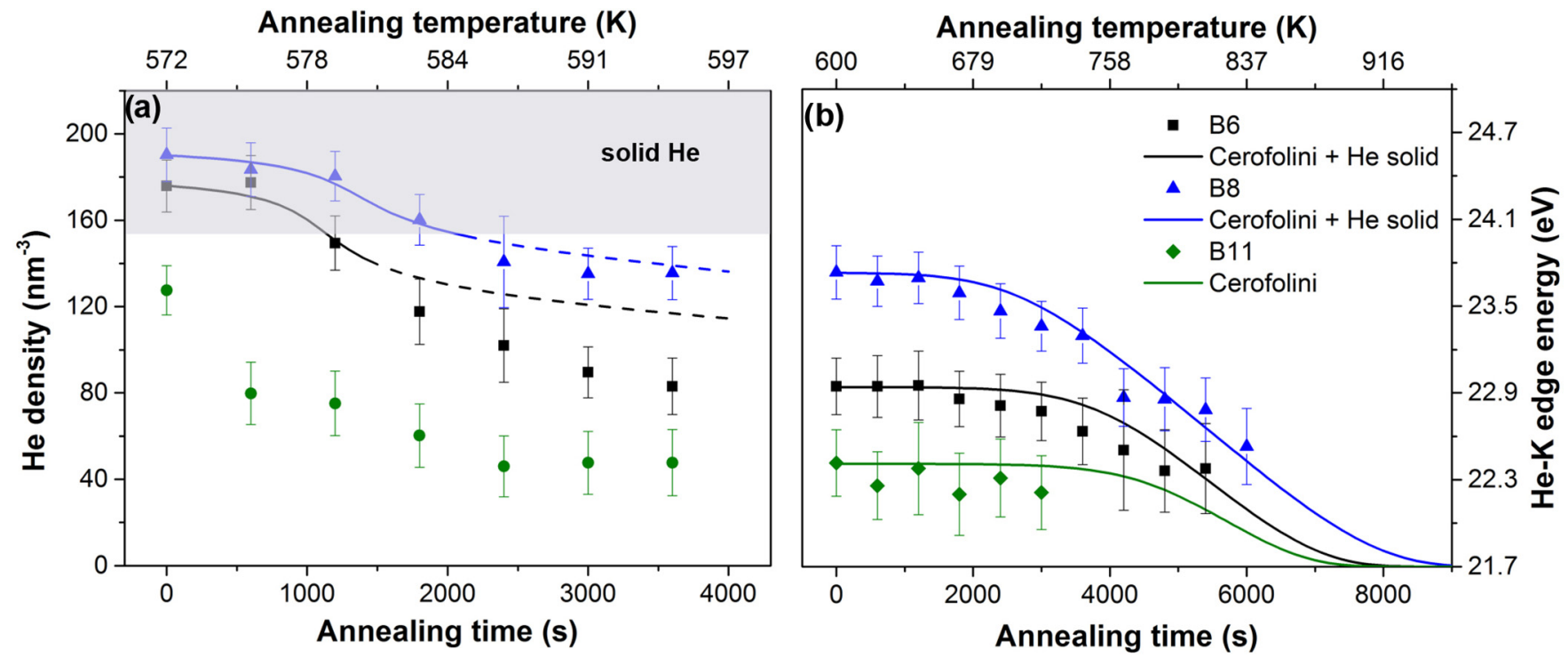

FIG. 8. Helium density measured in the center of bubbles B6, B8, and B11 in the S773K sample, together with the Cerofolini's model (b) and our solid helium model (a). The fitting parameters were $z=5 \mathrm{~nm}$ for each bubbles, $\zeta\left(n_{\mathrm{He}}\right)=2.021 \times 10^{-6} n_{\mathrm{He}}^{3}-7.437 \times 10^{-4} n_{\mathrm{He}}^{2}+$ $0.0888 n_{\mathrm{He}}-3.45$ for B6 and $\zeta\left(n_{\mathrm{He}}\right)=3.618 \times 10^{-6} n_{\mathrm{He}}^{3}-1.621 \times 10^{-3} n_{\mathrm{He}}^{2}+0.241 n_{\mathrm{He}}-11.92$ for B8.

step emission kinetic is poorly described using the Cerofolini's model, unlike the S773K sample. An important difference between $\mathrm{S} 973 \mathrm{~K}$ and S773K samples is the much higher initial helium density in most bubbles in the latter, in the range $170-180 \mathrm{He} \mathrm{nm}{ }^{-3}$. The equivalent pressure is in the range 20-24 GPa using the equation of states (E.O.S.) of Loubeyre et al. [58]. For such pressures, the phase transition between fluid and solid He occurs for temperatures in the range 450$490 \mathrm{~K}$ [59-62]. The measurement temperature being $473 \mathrm{~K}$, helium may be solid in the bubbles. The formation of rare gas bubbles in the crystalline state following the implantation of $\mathrm{Ar}, \mathrm{Kr}, \mathrm{Xe}$ in metals [63-71] and $\mathrm{Kr}$ and $\mathrm{Xe}$ in $\mathrm{Si}$ [72-75] is well documented in the literature. Moreover, the fluid to solid transition of He has been sporadically reported in bubbles in Al by positron lifetime and Doppler broadening measurements [76] and in $\mathrm{LiNbO}_{3}$ [77]. Assuming helium in a solid state at the beginning of the in situ annealing, it is hardly conceivable that the ultra dense fluid model would be suited for the whole kinetics. However, when the second step begins, the He density in the bubbles is in the range $80-150 \mathrm{He} \mathrm{nm}^{-3}$ for which the ultradense fluid model should apply. This is confirmed by the data shown in Fig. 8(b) where the emission kinetics of bubbles B6 and B8 (symbols), typical of all those observed, can be fairly fitted with the Cerofolini's model (full lines).

To model the first step, we develop an alternative model, accounting for solid helium in bubbles. In first approximation, we consider the contribution of vibration modes to the free energy of the solid helium, constituted of $N_{\mathrm{He}}$ vibrating helium atoms, and we assume that the vibration modes are similar in the three directions. The partition function of such a system is given by

$$
Z=\left(\frac{2}{\operatorname{sh}\left(\frac{h v}{2 k_{B} T}\right)}\right)^{3 N_{\mathrm{He}}}
$$

with $v$ the phonon frequency.
In the high-temperature approximation, which is justified here since the measurement temperature $T(473 \mathrm{~K})$ is much higher than the Debye temperature of solid He,

$$
Z=\left(\frac{k_{B} T}{h v}\right)^{3 N_{\mathrm{He}}} .
$$

Taking into account the potential $U$ with fcc solid He, the chemical potential is given by

$$
\begin{aligned}
\mu= & 3 k_{B} T \ln \left(\frac{h v}{k_{B} T}\right)+3 N_{\mathrm{He}} k_{B} T \frac{1}{v} \frac{\partial v}{\partial N_{\mathrm{He}}} \\
& +1 / 2 \omega u\left(k n_{\mathrm{He}}^{-1 / 3}\right) .
\end{aligned}
$$

In our model, it is important to consider the variation of the phonon frequency as a function of the helium density. For the densities considered here, the high pressure data by Watson et al. [78] can be approximated by a linear equation

$$
v=a n_{\mathrm{He}}-v_{0}
$$

with $a=6 \times 10^{10} \mathrm{~cm}^{-3} \mathrm{~s}^{-1}$ and $\nu_{0}=3 \times 10^{12} \mathrm{~s}^{-1}$.

As in the Cerofolini's model, assuming equilibrium between the lattice gas and the solid phase in the bubble implies that the chemical potentials of both assembly of atoms are equal. This allows for the calculation of the helium density in the bubble as a function of the helium density in the matrix. The rate equation is then given by

$$
\begin{aligned}
-\frac{d n_{\mathrm{He}}}{d t}= & 2 \frac{D_{0} c_{s} \lambda_{T}^{3}}{V z}\left(\frac{\exp \left(-h v_{i} / 2 k_{B} T\right)}{1-\exp \left(-h v_{i} / k_{B} T\right)}\right)^{3} \\
& \times \exp \left(-\frac{E^{\mathrm{act}}+\zeta\left(n_{\mathrm{He}}\right)-1 / 2 \omega u\left(k n_{\mathrm{He}}^{-1 / 3}\right)}{k_{B} T}\right) \\
& \times \exp \left(3 \ln \left(\frac{h v}{k_{B} T}\right)+3 n_{\mathrm{He}} \frac{a}{a n_{\mathrm{He}}-v_{0}}\right) .
\end{aligned}
$$


In Eq. (7), we introduced $\zeta\left(n_{\mathrm{He}}\right)$ as a correction to $E^{\text {act }}$, the energy barrier for an helium atom to be emitted from the bubble. $\zeta\left(n_{\mathrm{He}}\right)$ accounts for additional contributions because of the very high helium pressure in bubbles, and helium in solid state. First, there might be a supplementary energy barrier associated to the diffusion of helium atoms through the bubble internal surfaces. Firs-principles calculations have shown the existence of low energy migration paths [52]. Since helium atoms in a fluid state are characterized by a high mobility and no stationary position relative to the surface, it is assumed that they can easily find and follow these paths. Consequently, there should be no additional energy contributions for helium emission from the bubble. Conversely, helium atoms in solid state have low mobility and the diffusion through easy paths will be limited, leading to an overall higher energy barrier for diffusion. Moreover, the bubbles aspect ratio is broadly distributed from 1.2 to 1.9 , indicating different proportions of surface orientations. The additional energy barrier represented by $\zeta\left(n_{\mathrm{He}}\right)$ could then vary from one bubble to another. Secondly, our investigations recently revealed the formation of an highly pressurized region in the silicon matrix, surrounding the bubble, when the helium density in the bubble is greater than $114 \mathrm{He} \mathrm{nm}^{-3}$ [41]. This is expected to impede the diffusion of $\mathrm{He}$ atoms, and thus also the helium emission from the bubble. All these factors may contribute to $\zeta\left(n_{\mathrm{He}}\right)$, and thus to an increase of the activation energy associated to helium emission from the bubbles. This argument is supported by earlier desorption experiments on Ar bubbles in $\mathrm{Ni}$, where an anomalous desorption peak as been ascribed to the phase transformation of Ar inside the bubbles [71].

The results of our calculations are shown in Fig. 8(a). $z$ is set using the Cerofolini's fit on the data in the range 600 to $900 \mathrm{~K}$ (second helium emission mechanism) and $\zeta\left(n_{\mathrm{He}}\right)$ is adjusted by a third order polynomial function to reproduce the experimental data; its value decreases from $0.2 \mathrm{eV}$ to $0.0 \mathrm{eV}$ during the helium emission. As seen, this model succeeds in reproducing the experimental data of the first annealing step, when helium is assumed to be solid (Fig. 8).

As for bubbles B2, B9, and B11, the second part of their emission kinetics is well described by the Cerofolini's model but the first part can not be described neither by our solid $\mathrm{He}$ model (for a helium density of $150 \mathrm{He} \mathrm{nm}^{-3}$, helium is probably not solid [58]) nor by the Cerofolini's model. For the moment, the reasons for this anomalous behavior are not clear and further experiments are needed.

\section{DISCUSSION}

Helium emission from bubbles has been mainly studied in the literature by THDS. The THDS spectra of highfluence helium implanted samples are usually characterized by two peaks. A first one at low temperatures (600-900 K) is attributed to helium emission from small helium-vacancies clusters, whereas the second one, occurring at 400 or $500 \mathrm{~K}$ higher temperatures is attributed to helium emission from bubbles [20,24,29]. We do not measure the first peak using in situ EFTEM-SI because the small He-V clusters can not be resolved. However, we clearly demonstrate here that the helium emission from individual bubbles can be measured. In agreement with previous investigations by THDS, our results show that when the helium density in the bubble is below 150 $\mathrm{He} \mathrm{nm}^{-3}$, helium emission is associated to a single physical mechanism, with an activation energy of $1.8 \mathrm{eV}$ [23,24,52]. This mechanism can be modelled using the ultradense fluid Cerofolini's model, thus proving that more simple perfect gas or van der Waals descriptions are inappropriate. The temperatures at which we observe helium emission from bubbles can however be surprising. They are indeed much smaller than what is usually obtained by THDS. This is in part ascribed to the different temperature ramps used in both experiments. Indeed, the typical ramps used for THDS measurements are typically one or two order of magnitude larger than what we used here, and it is well known that the temperature ramp plays an important role in the helium release process $[35,36]$. Moreover, as seen, the position of the bubble with respect to the free surfaces greatly influences the emission kinetics. Both effects suggest that the combination of structural data obtained through in situ TEM, thus on thin foils, and of chemical data obtained by ex situ THDS on bulk samples, should be done with great care.

When the initial helium density is higher, we have shown that the Cerofolini's model can no longer reproduce the helium emission kinetics. We ascribe this to the fact that helium may be in a solid state and that there are additional barriers for helium emission associated with high internal pressures. We propose a tentative model taking into account these aspects, and which can model our experiments with a fair agreement. The influence of high pressures on helium emission from the bubbles is represented by the contribution $\zeta\left(n_{\mathrm{He}}\right)$, which correctly decreases as a function of $n_{\mathrm{He}}$ and becomes negligible for $150 \mathrm{He} \mathrm{nm}^{-3}$. Also, the maximum value of $0.2 \mathrm{eV}$ found for the highest densities is reasonable in the light of the proposed mechanisms. Although this value is low, we show that it strongly influences the helium emission kinetics.

In this work, we monitored the morphology and position of single nanometric bubbles as a function of temperature, while measuring the helium density inside these bubbles. These original experiments allow for an improved understanding compared to the current state of the art. Two qualitatively different mechanisms are generally distinguished to explain cavity coarsening upon annealing, namely cavity migration and coalescence and Ostwald Ripening. The former mechanism is due to random rearrangements of the cavity matrix interface by diffusion of matrix atoms by surface diffusion whereas in the latter mechanism, the thermally activated resolution of helium and vacancies from small bubbles leads to the absorption by larger ones. We found here that helium emission takes place at temperatures for which no bubble migration is seen. Conversely, at temperatures higher than $900 \mathrm{~K}$, several migration events are detected, leading to either loss of bubbles at the surface or to coalescence $(\mathrm{B} 3+\mathrm{B} 4)$. This first indicates that the observations of mobile bubbles made at higher temperatures actually concerned empty, i.e., voids, or weakly filled cavities. This confirms with no ambiguities previous observations by Griffioen et al. [21] or more recently by Dumont et al. [31]. Moreover, this is in line with the theoretical work of Evans who has shown that for moderate annealing temperature up to $1273 \mathrm{~K}$, the coarsening of voids is due to migration and coalescence [28]. The migration of voids is attributed to a surface diffusion process. This is supported for instance by the 
fact that the presence of oxygen impedes any voids coarsening [25]. In the migration and coalescence model, smaller bubbles are more mobile than larger ones for a given temperature or in other words, smaller bubbles are expected to migrate at a lower temperature than larger ones [27]. The migration of the $S$ voids is visible at about $920 \mathrm{~K}$, the migration of the $L$ ones would thus be expected to occur at higher temperatures. This is indeed the case for B10 and B6, $10 \mathrm{~nm}$ in diameter, which migrate at $970 \mathrm{~K}$ and for $\mathrm{B} 5$ and $\mathrm{B} 9,12 \mathrm{~nm}$ in diameter at $1050 \mathrm{~K}$. Because of the small thickness of the thin TEM foil, the largest migrating voids often burst out at the surface before meeting other voids, explaining why in average the diameter of the largest voids does not change. However, as expected, the diameter of the smaller ones clearly increases during annealing at temperatures higher than $920 \mathrm{~K}$ whereas the cavity density decreases.

The lack of mobility of bubbles containing a high helium density has two possible explanations. First, it is usually assumed that bubbles migrate thanks to the diffusion of matrix atoms on the bubble internal surfaces. The presence of helium, especially at high density, is expected to impede this mechanism, thus greatly lowering the bubble mobility [79]. The modification of bubble aspect ratio at constant diameter is also expected to depend on surface diffusion. In fact, we note that in most of the bubbles in both samples, with aspect ratio in the range 1 to 1.5 , the bubbles morphology does not change. However, B9 in the S773K sample, exhibiting an initial higher aspect ratio of 1.8 , tends to spherical shape when annealed, suggesting that a limited surface diffusion is active. Second, it is also possible that for the bubble sizes and helium densities studied here, the diffusion starts for temperatures higher than the ones associated with helium emission.

We now focus on the formation of these nanometric sized bubbles filled with a high helium density, such as those observed in S773K before in situ annealing. We know that Ostwald ripening is unlikely to occur for such temperatures [28], especially because of the high vacancy formation energy $(3.17-3.6 \mathrm{eV}[80,81])$ [82]. This is confirmed in our experiments, where no helium exchange between bubbles and no bubble shrinkage are detected. In a scenario compatible with our results, bubble coarsening could then occur thanks to the migration of sub-nanometric sized bubbles, hardly detectable in experiments. These mobile bubbles, filled by a low helium density, would then coalesce with larger and less mobile bubbles. This would explain the formation of bubbles of diameter 10-20 $\mathrm{nm}$. Nevertheless, this process can not explain the high helium density inside the large bubbles, as measured here. Instead we propose that the helium density increase is the consequence of the capture of helium interstitials, mobile at moderate temperatures. Such a mechanism is supported by molecular dynamics investigations performed in our group $[41,83]$.

\section{CONCLUSION}

In this work, we have studied the detail of the evolution of single nanometric helium bubbles by simultaneously monitoring their structural and chemical modifications during an in situ annealing in the TEM. We clearly show that helium emission occurs at temperatures for which no motion of bubbles is observed. At higher temperatures, the migration (and coalescence) of voids is clearly revealed. For helium density lower than $150 \mathrm{He} \mathrm{nm}^{-3}$, the Cerofolini's model taking into account the thermodynamical properties of an ultradense fluid reproduces well the helium emission from the bubbles leading to an activation energy of $1.8 \mathrm{eV}$. When bubbles exhibit a higher initial helium density, the Cerofolini's model fails to reproduce the helium emission kinetics. We ascribe this to the fact that helium may be in the solid phase and we propose a tentative model to take into account the properties of the solid.

\section{ACKNOWLEDGMENTS}

D. T. L. Alexander (CIME, Lauzanne) is kindly acknowledged for the technical help on the microscope. K.A. acknowledges the French doctoral school SI-MMEA for financial support.
[1] C. Herman, Cool as helium, Nat. Chem. 4, 140 (2012).

[2] Fundamental Aspects of Inert Gases in Solids, edited by S. E. Donnelly and J. H. Evans (Plenum Press, New York, 1991).

[3] T. Sato, N. Funamori, and T. Yagi, Helium penetrates into silica glass and reduces its compressibility, Nat. Commun. 2, 345 (2011).

[4] J. A. Sans, F. J. Manjón, C. Popescu, V. P. Cuenca-Gotor, O. Gomis, A. Muñoz, P. Rodríguez-Hernández, J. ContrerasGarcía, J. Pellicer-Porres, A. L. J. Pereira, D. SantamaríaPérez, and A. Segura, Ordered helium trapping and bonding in compressed arsenolite: Synthesis of $\mathrm{As}_{4} \mathrm{O}_{6} \cdot 2 \mathrm{He}$, Phys. Rev. B 93, 054102 (2016).

[5] H. Trinkaus and B. N. Singh, Helium accumulation in metals during irradiation - where do we stand? J. Nucl. Mater. 323, 229 (2003); Proceedings of the Second \{IEA\} Fusion Materials Agreement Workshop on Modeling and Experimental Validation.
[6] S. Takamura, N. Ohno, D. Nishijima, and S. Kajita, Formation of nanostructured tungsten with arborescent shape due to helium plasma irradiation, Plasma Fusion Res. 1, 051 (2006).

[7] N. Li, E. G. Fu, H. Wang, J. J. Carter, L. Shao, S. A. Maloy, A. Misra, and X. Zhang, He ion irradiation damage in $\mathrm{Fe} / \mathrm{W}$ nanolayer films, J. Nucl. Mat. 389, 233 (2009).

[8] M. J. Demkowicz, D. Bhattacharyya, I. Usov, Y. Q. Wang, M. Nastasi, and A. Misra, The effect of excess atomic volume on He bubble formation at fcc-bcc interfaces, Appl. Phys. Lett. 97, 161903 (2010).

[9] G. R. Odette and D. T. Hoelzer, Irradiation-tolerant nanostructured ferritic alloys: Transforming helium from a liability to an asset, JOM 62, 84 (2010).

[10] N. Li, J. J. Carter, A. Misra, L. Shao, H. Wang, and X. Zhang, The influence of interfaces on the formation of bubbles in He-ion-irradiated Cu/Mo monolayers, Phil. Mag. Lett. 91, 18 (2011). 
[11] M. Bruel, Silicon on insulator material technology, Electron. Lett. 31, 1201 (1995).

[12] D. Alquier, C. Bongiorno, F. Roccaforte, and V. Raineri, Interaction between dislocations and He-implantation-induced voids in GaN epitaxial layers, Appl. Phys. Lett. 86, 211911 (2005).

[13] B. Hollander, S. T. Lenk, S. Mantl, H. Trinkaus, D. Kirch, M. Luysberg, T. Hakbarth, H. J. Herzog, and P. F. P. Fichtner, Strain relaxation of pseudomorphic $\mathrm{Si}_{1-x} \mathrm{Ge}_{x} / \mathrm{Si}(100)$ heterostructures after hydrogen or helium ion implantation for virtual substrate fabrication, Nucl. Instrum. Methods Phys. Res. B 175-177, 357 (2001).

[14] G. A. Petersen, S. M. Myers, and D. M. Follstaedt, Gettering of transition metals by cavities in silicon formed by helium ion implantation, Nucl. Inst. Methods Phys. Res. B 127/128, 301 (1997).

[15] X. Ou, R. Kögler, A. Mücklich, W. Skorupa, W. Möller, X. Wang, and L. Vines, The use of nanocavities for the fabircation of ultrathin buried oxide layers, Appl. Phys. Lett. 94, 011903 (2009).

[16] V. Godinho, J. Caballero-Hernández, D. Jamon, T. C. Rojas, R. Schierholz, J. García-López, F. J. Ferrer, and A. Fernández, A new bottom-up methodology to produce silicon layers with a closed porosity nanostructure and reduced refractive index, Nanotechnology 24, 275604 (2013).

[17] S. Kajita, T. Yoshida, D. Kitaoka, R. Etoh, M. Yajima, N. Ohno, H. Yoshida, N. Yoshida, and Y. Terao, Helium plasma implantation on metals: Nanostructure formation and visiblelight photocatalytic response, J. Appl. Phys. 113, 134301 (2013).

[18] S. Iyyakkunnel, L. Marot, B. Eren, R. Steiner, L. Moser, D. Mathys, M. Düggelin, P. Chapon, and E. Meyer, Morphological changes of tungsten surfaces by low-flux helium plasma treatment and helium incorporation via magnetron sputtering, ACS Appl. Mater. Interfaces 6, 11609 (2014).

[19] R. Schierholz, B. Lacroix, V. Godinho, J. Caballero-Hernández, M. Duchamp, and A. Fernández, STEM-EELS analysis reveals stable high-density $\mathrm{He}$ in nanopores of amorphous silicon coatings deposited by magnetron sputtering, Nanotechnology 26, 075703 (2015).

[20] G. F. Cerofolini, F. Corni, S. Frabboni, C. Nobili, G. Ottaviani, and R. Tonini, Hydrogen and helium bubbles in silicon, Mater. Sci. Eng. A 27, 1 (2000).

[21] C. C. Griffioen, J. H. Evans, P. C. de Jong, and A. van Veen, Helium desorption permeation from bubbles in silicon: a novel method of void production, Nucl. Instrum. Methods Phys. Res. B 27, 417 (1987).

[22] P. J. Goodhew and S. K. Tyler, Helium bubble behaviour in b. c. c. metals below $0.65 \mathrm{~T}_{\mathrm{m}}$, Proc. Royal Soc. A 377, 151 (1981).

[23] S. Godey, E. Ntsoenzok, T. Sauvage, A. van Veen, F. Labohm, M. F. Beaufort, and J. F. Barbot, Helium desorption from cavities induced by high energy ${ }^{3} \mathrm{He}$ and ${ }^{4} \mathrm{He}$ implantation in silicon, Mater. Sci. Eng. B 73, 54 (2000).

[24] E. Oliviero, M. L. David, MF Beaufort, J. F. Barbot, and A. van Veen, On the effect of implantation temperature in helium implanted silicon, Appl. Phys. lett. 81, 4201 (2002).

[25] J. H. Evans, A. Van Veen, and C. C. Griffioen, The annealing of helium-induced cavities in silicon and the inhibiting role of oxygen, Nucl. Instrum. Methods Phys. Res., Sect. B 28, 360 (1987).
[26] D. M. Follstaedt, S. M. Myers, G. A. Petersen, and J. W. Medernach, Cavity formation and impurity gettering in Heimplanted Si, J. Electron. Mat. 25, 157 (1996).

[27] S. E. Donnelly, V. M. Vishnyakov, R. C. Birtcher, and G. Carter, The effects of radiation damage and impurities on void dynamics in silicon, Nucl. Ins. Methods. Phys. Res. B 175-177, 132 (2001).

[28] J. H. Evans, Mechanisms of void coarsening in helium implanted silicon, Nucl. Ins. Methods. Phys. Res. B 196, 125 (2002).

[29] S. Frabboni, F. Corni, C. Nobili, R. Tonini, and G. Ottaviani, Nanovoid formation in helium-implanted single-crystal silicon studied by in situ techniques, Phys. Rev. B 69, 165209 (2004).

[30] D. Babonneau, M.-F. Beaufort, A. Declemy, J.-F. Barbot, and J.-P. Simon, Grazing incidence small-angle x-ray scattering from defects induced by helium implantation in silicon, J. Appl. Phys. 99, 113507 (2006)

[31] M. Dumont, G. Regula, M.-V. Coulet, M.-F. Beaufort, E. Ntsoenzok, and B. Pichaud, Growth and migration of nanocavities in $\mathrm{He}^{+}$multi-implanted si measured by in situ small-angle x-ray scattering, Mater. Sci. Eng. B 182, 45 (2014).

[32] V. Raineri, M. Saggio, and E. Rimini, Voids in silicon by $\mathrm{He}$ implantation: from basic to applications, J. Mater. Res. 15, 1449 (2000).

[33] E. Oliviero, M. F. Beaufort, and J. F. Barbot, Influence of dose rate on bubble formation by high energy $\mathrm{He}$ implantation in silicon, J. Appl. Phys. 90, 1718 (2001).

[34] M. L. David, M. F. Beaufort, and J. F. Barbot, Effect of implant temperature on defects created using high fluence of helium in silicon, J. Appl. Phys. 93, 1438 (2003).

[35] T. W. Simpson and I. V. Mitchell, Effect of annealing temperature ramp rate on bubble formation in helium-implanted silicon, Appl. Phys. Lett. 86, 241907 (2005).

[36] F. Roqueta, A. Grob, J. J. Grob, R. Jérisian, J. P. Stoquert, and L. Ventura, Stability of cavities formed by He implantation in silicon, Nucl. Instrum. Methods Phys. Res., Sect. B 147, 298 (1999).

[37] B. S. Li, C. H. Zhang, Y. R. Zhong, D. N. Wang, L. H. Zhou, Y. T. Yang, L. Q. Zhang, H. H. Zhang, Y. Zhang, and L. H. Han, Annealing ambient on the evolution of He-induced voids in silicon, Appl. Surf. Sci. 257, 7036 (2011).

[38] M.-L. David, F. Pailloux, V. Mauchamp, and L. Pizzagalli, In situ probing of helium desorption from individual nanobubbles under electron irradiation, Appl. Phys. Lett. 98, 171903 (2011).

[39] M.-L. David, K. Alix, F. Pailloux, V. Mauchamp, M. Couillard, G. A. Botton, and L. Pizzagalli, In situ controlled modification of the helium density in single helium-filled nanobubbles, Journ. Appl. Phys. 115, 123508 (2014).

[40] K. Alix, M.-L. David, G. Lucas, D. T. L. Alexander, F. Pailloux, C. Hébert, and L. Pizzagalli, Gentle quantitative measurement of helium density in nanobubbles in silicon by spectrum imaging, Micron 77, 57 (2015).

[41] J. Dérès, M.-L. David, K. Alix, C. Hébert, D. T. L. Alexander, and L. Pizzagalli, Properties of helium bubbles in covalent systems at the nanoscale: A combined numerical and experimental study, Phys. Rev. B 96, 014110 (2017).

[42] J. C. Rife, S. E. Donnelly, A. A. Lucas, J. M. Gilles, and J. J. Ritsko, Optical Absorption and Electron-Energy-Loss Spectra of Helium Microbubbles in Aluminum, Phys. Rev. Lett. 46, 1220 (1981).

[43] A. A. Lucas, J. P. Vigneron, S. E. Donnelly, and J. C. Rife, Theoretical interpretation of the vacuum ultraviolet reflectance 
of liquid helium and of the absorption spectra of helium microbubbles in aluminum, Phys. Rev. B 28, 2485 (1983).

[44] H. G. Kuhn, Atomic Spectra (Academic Press, New York, 1962), p. 132.

[45] D. Taverna, M. Kociak, O. Stéphan, A. Fabre, E. Finot, B. Décamps, and C. Colliex, Probing Physical Properties of Confined Fluids within Individual Nanobubbles, Phys. Rev. Lett. 100, 035301 (2008).

[46] S. Frechard, M. Walls, M. Kociak, J. P. Chevalier, J. Henry, and D. Gorse, Study by EELS of helium bubbles in a martensitic steel, J. Nucl. Mat. 393, 102 (2009).

[47] D. Babonneau, D. Lantiat, S. Camelio, J. Toudert, L. Simonot, F. Pailloux, M.-F. Denanot, and T. Girardeau, Gold and silver nanoparticles embedded in dielectric-capping layers studied by HAADF-STEM, Eur. Phys. J. Appl. Phys. 44, 3 (2008).

[48] D. J. Eaglesham, A. E. White, L. C. Feldman, N. Moriya, and D. C. Jacobson, Equilibrium Shape of Si, Phys. Rev. Lett. 70, 1643 (1993).

[49] See Supplemental Material at http://link.aps.org/supplemental/ 10.1103/PhysRevB.97.104102 for a movie of the in situ annealing of the S773K sample.

[50] G. F. Cerofolini, G. Calzolari, F. Corni, S. Frabboni, C. Nobili, G. Ottaviani, and R. Tonini, Thermal desorption spectra from cavities in helium-implanted silicon, Phys. Rev. B 61, 10183 (2000).

[51] A. Charaf Eddin, G. Lucas, M. F. Beaufort, and L. Pizzagalli, DFT calculation of the stability and mobility of noble gas atoms in silicon, Comput. Mater. Sci. 44, 1030 (2009).

[52] A. Charaf Eddin and L. Pizzagalli, First-principles calculations of helium and neon desorption from cavities in silicon, J. Phys.: Condens. Matter 24, 175006 (2012).

[53] G. F. Cerofolini, G. Calzolari, F. Corni, C. Nobili, G. Ottaviani, and R. Tonini, Ultradense gas bubbles in hydrogen- or heliumimplanted (or coimplanted) silicon, Mater. Sci. Eng. B 71, 196 (2000).

[54] F. Ziegler, J. B. Biersack, and U. Littmark, The Stopping Range of Ions in Solids (Pergamon, New York, 1985); http://www.srim.org.

[55] F. Pailloux, M.-L. David, and L. Pizzagalli, Quantitative HRTEM investigation of nanoplatelets, Micron 41, 135 (2010).

[56] M. Alatalo, M. J. Puska, and R. M. Nieminen, First-principles study of He in Si, Phys. Rev. B 46, 12806 (1992).

[57] M. A. Nguyen, M.-O. Ruault, and F. Fortuna, Formation and growth of nanocavities and cavities induced by $\mathrm{He}^{+}$implantation in silicon, Adv. Nat. Sci.: Nanosci. Nanotechnol. 3, 015015 (2012).

[58] P. Loubeyre, R. LeToullec, J. P. Pinceaux, H. K. Mao, J. Hu, and R. J. Hemley, Equation of State and Phase Diagram of Solid ${ }^{4} \mathrm{He}$ from Single-Crystal x-ray Diffraction Over a Large $P-T$ Domain, Phys. Rev. Lett. 71, 2272 (1993).

[59] A. Driessen, E. van der Poll, and I. F. Silvera, Equation of state of solid ${ }^{4} \mathrm{He}$, Phys. Rev. B 33, 3269 (1986).

[60] W. L. Vos, M. G. E. van Hinsberg, and J. A. Schouten, Highpressure triple point in helium: The melting line of helium up to 240 kbar, Phys. Rev. B 42, 6106 (1990).

[61] F. Datchi, P. Loubeyre, and R. LeToullec, Extended and accurate determination of the melting curves of argon, helium, ice $\left(\mathrm{H}_{2} \mathrm{O}\right)$, and hydrogen $\left(\mathrm{H}_{2}\right)$, Phys. Rev. B 61, 6535 (2000).

[62] D. Santamarìa-Pérez, G. D. Mukherjee, B. Schwager, and R. Boehler, High-pressure melting curve of helium and neon: deviations from corresponding states theory, Phys. Rev. B 81, 214101 (2010).

[63] C. Templier, C. Jaouen, J. P. Riviére, J. Delafond, and J. C. R. Grilhé, Précipitation du Xénon implanté dans des alliages d'aluminium, C. R. Acad. Sci. Paris 299, 613 (1984).

[64] A. vom Felde, J. Fink, Th. Müller-Heinzerling, J. Pflüger, B. Scheerer, G. Linker, and D. Kaletta, Pressure of Neon, Argon, and Xenon Bubbles in Aluminum, Phys. Rev. Lett. 53, 922 (1984).

[65] J. H. Evans and D. J. Mazey, Evidence for solid krypton bubbles in copper, nickel and gold at 293 K, J. Phys. F 15, 21 (1985).

[66] J. H. Evans and D. J. Mazey, The formation of solid krypton bubbles in molybdenum, Scr. Metall. 19, 621 (1985).

[67] S. E. Donnelly and C. J. Rossouw, Lattice images of inert gas bubbles in aluminium, Nucl. Ins. Methods. Phys. Res. B 13, 485 (1986).

[68] C. Templier, H. Garem, J. C. Desoyer, and J. Delafond, Xe+Kr and $\mathrm{Xe}+\mathrm{Ar}$ bubbles in implanted aluminium, Scr. Metall. 20, 1705 (1986).

[69] I. Hashimoto, H. Yorikawa, H. Mitsuya, H. Yamaguchi, K. Takaishi, T. Kikuchi, K. Furuya, E. Yagi, and M. Iwaki, Annealing behavior of krypton-implanted aluminum, J. Nucl. Mater. 149, 69 (1987).

[70] R. C. Birtcher and W. Jaeger, Precipitation and phase stability of solid $\mathrm{Kr}$ in cavities after room-temperature implantation of $\mathrm{Al}$, Ultramicroscopy 22, 267 (1987).

[71] D. B. Kuzminov and V. N. Chernikov, in Fundamental Aspects of Inert Gases in Solids, edited by S. E. Donnelly and J. H. Evans (Plenum Press, New York, 1991), p. 337.

[72] C. Templier, B. Boubeker, H. Garem, E. L. Mathé, and J. C. Desoyer, Precipitation of implanted xenon in silicon, Physica Status Solidi (a) 92, 511 (1985).

[73] G. Faraci, A. R. Pennisi, A. Terrasi, and S. Mobilio, Xe bubbles in si observed by extended x-ray-absorption fine-structure spectroscopy, Phys. Rev. B 38, 13468 (1988).

[74] G. Faraci, A. R. Pennisi, and J.-L. Hazemann, Xanes of highpressure $\mathrm{Kr}$ clusters in Be and Se, Phys. Rev. B 56, 12553 (1997).

[75] G. Faraci, A. R. Pennisi, F. Zontone, B. Li, and I. Petrov, Expansion and melting of Xe nanocrystals in Si, Phys. Rev. B 74, 235436 (2006).

[76] S. Maji, A. Singh, and P. M. G. Nambissan, Solid phase transition of overpressurised helium bubbles seen from positron annihilation studies, Phys. Lett. A 281, 76 (2001).

[77] A. Ofan, L. Zhang, O. Gaathon, S. Bakhru, H. Bakhru, Y. Zhu, D. Welch, and R. M. Osgood, Spherical solid he nanometer bubbles in an anisotropic complex oxide, Phys. Rev. B 82, 104113 (2010).

[78] G. H. Watson and W. B. Daniels, Raman scattering from solid helium at high pressure, Phys. Rev. B 31, 4705 (1985).

[79] E. YA. Mikhlin, Suppression of diffusion mobility of small gas bubbles in solids, Physica Status Solidi (a) 56, 763 (1979).

[80] S. Goedecker, T. Deutsch, and L. Billard, A Fourfold Coordinated Point Defect in Silicon, Phys. Rev. Lett. 88, 235501 (2002).

[81] S. Dannefaer, P. Mascher, and D. Kerr, Monovacancy Formation Enthalpy in Silicon, Phys. Rev. Lett. 56, 2195 (1986).

[82] J. H. Evans, Fundamental Aspects of Inert Gases in Solids (Plenum Press, New York, 1991).

[83] L. Pizzagalli, M. L. David, and M. Bertolus, Molecular dynamics simulation of the initial stages of he bubbles formation in silicon, Modell. Simul. Mater. Sci. Eng. 21, 065002 (2013). 